\title{
Evolution of Surgery Offered to Aspergilloma over 2 Decades, Institutional Report
}

\author{
Hysam Abdelmohty ${ }^{1,}$, , Salah Eldin Khalaf ${ }^{1}$, Walid Hassan ${ }^{1}$, Ahmed Mostafa ${ }^{1}$, \\ Mohamed-Adel Elanwar ${ }^{2}$ \\ ${ }^{1}$ Cardiothoracic Surgery Department, Mansoura University, Mansoura, Egypt \\ ${ }^{2}$ Cardiothoracic Surgery Department, Cairo University, Cairo, Egypt
}

\section{Email address:}

hysam.mohty@gmail.com (H. Abdelmohty)

${ }^{*}$ Corresponding author

\section{To cite this article:}

Hysam Abdelmohty, Salah Eldin Khalaf, Walid Hassan, Ahmed Mostafa, Mohamed-Adel Elanwar. Evolution of Surgery Offered to Aspergilloma over 2 Decades, Institutional Report. International Journal of Cardiovascular and Thoracic Surgery.

Vol. 6, No. 5, 2020, pp. 60-65. doi: 10.11648/j.ijcts.20200605.12

Received: October 16, 2020; Accepted: October 26, 2020; Published: November 9, 2020

\begin{abstract}
Background: Aspergilloma is the localized form of pulmonary colonization by Aspergillus species, which often hosted within a pre-existing cavitary lesion. The Aspergilloma (commonly known as mycetoma or fungus ball) consists of fungal hyphae, aggregates of inflammatory cells, fibrin threads, and destructed tissues debris. Aspergillus Fumigatu is the commonest species responsible for such lesions. In this study, over 20 years, data were collected for examination, on the outcomes of surgery for pulmonary Aspergilloma. Methods: Retrospective study of 54 patients, dating from January 1996 and December, 2015. Each patient's preoperative, diagnostic, operative, postoperative and follow up data was collected for analysis. Results: Findings of 54 patients who underwent surgery for Aspergilloma, 47 had clinical diagnosis. While remaining 7 were confirmed post-resection. The median age was 46.3 7.8 (aged 17-64 years). Risk assessment identified that greater probability for the Left Lung to be infected and increase if gender was male (2.6:1). The main presentation was hemoptysis, seen in $70.4 \%$ of cases, while symptoms of cough and expectoration occurred in $83.3 \%$ of them. The most prevalent predisposing factor was tuberculosis (TB), present in $57.4 \%$ of cases. The indication for surgery was recurrent hemoptysis, asymptomatic simple Aspergilloma and complex Aspergilloma. All the patients underwent pulmonary resection, with $82.5 \%$ of cases having lobectomy. The main postoperative complication was prolonged air leak $29.6 \%$ (16/54). The in-hospital mortality rate was $7.5 \%(6 / 54)$ patients. Conclusion: Surgery offered to Aspergilloma patients (fungus ball) brought beneficial results with an acceptable morbidity. The mortality observed within these cases, was predominantly due to high risk patients, with complex Aspergillosis. Recommendations for a multidisciplinary approach, in future cases, are paramount for better selection criteria.
\end{abstract}

Keywords: Fungus Ball, Hyphae, Aspergilloma, Cavity, Hemoptysis, Lobectomy

\section{Introduction}

Aspergillus encompasses over 350 known species. Pulmonary forms of Aspergillus affection include 'Aspergilloma'; the localized form. Aspergillus Fumigatu is the commonest species responsible for such lesions. Another form; allergic bronchopulmonary Aspergillosis which represents immunologic response to non-invasive species, while the 'disseminated Aspergillosis' is well recognized as a life-threatening infection in immunocompromised patients, both acquired and inherited or under chronic immunosuppression therapy mostly with steroids. In such cases, the pathology is multicenteric and the surgical option is limited. Aspergillosis is the third cause of hospitalization due to fungal infection. In our study, we analyzed the outcome of surgical intervention in patients with Aspergilloma. [1-3]

\section{Patients and Methods}

\subsection{Study Population}

In this retrospective report encompassing a homogenous 
cohort of 54 cases, over 20 years' period, data on the surgery offered and its outcomes for pulmonary Aspergilloma, at cardiothoracic surgery department, from the respected Cardiothoracic surgery department at Mansoura University Hospitals.

\subsection{Data Collection}

Through two decades interval from January 1996 and December 2015, an extensive review of the available data and records within our department was undertaken. Demographic data like age, sex and clinical data like symptoms, lobe involved and type of surgery and the results of surgical therapy, both morbidity and mortality, were analyzed. Radiologic examination data together with pathological findings were our keys for recruitment. Chest xray, computed tomography (CT) chest, fiber-optic bronchoscopy and bronchial lavage culture were routinely done.

\subsection{CT Radiologic Signs}

Our major radiological signs were:

Nodule: rounded opacity less than $3 \mathrm{~cm}$ in diameter.

Mass: round opacity greater than $3 \mathrm{~cm}$ in diameter.

Patch: opacity with irregular edges but cannot exceed a segment.

Massive consolidation: opacity in segments or lobes distribution, with air bronchogram inside. [4, 12]

Wedge shaped consolidation: broad base opacity and apex toward the lung hilum.

Cavity: radiolucency within a nodular area or mass consolidation.

Halo sign: mass surrounded by a zone of lower attenuation.

Atelectasis: lobar or segmental opacity with shrinking of the tissue volume.

Pleural effusion: a high density opacity obliterating the costophernic angle with rising level towards the Axilla. [4, 12]

\subsection{Histochemical Diagnosis}

Patients were assigned with a proven diagnosis of pulmonary Aspergilloma if histological tissue examination revealed septated, branching fungi upon retrieval of any Aspergillus species from pulmonary tissues or positivity in immunohistochemical detection of anti-Aspergillus monoclonal antibody. Also, mycological evidence by culture or cytological analysis of bronchoalveolar lavage fluid.

\subsection{Protocol for Surgery}

Intravenous Amphotricen B was given for at least one week to sterilize the field except in true emergencies. Followup of the liver and kidney functions and Potassium level was mandatory with anti-fungal therapy. If the patient was on chemotherapy, 4 weeks interval of was mandatory and strictly followed except in cases of massive hemoptysis. Conventional lung resection was done for all patients using single lung ventilation. Postoperative pain control using both Pethidine and non-steroidal anti-inflammatory drugs as needed. Postoperative complications were reported and managed accordingly. Follow-up in both thoracic surgery and chest medicine clinics was done monthly for most of the survivors. Clinical examination, chest $\mathrm{x}$-ray and complete blood picture were routinely done in our follow-up.

\section{Statistical Analysis}

The data extracted was tabulated out using Microsoft Excel (Microsoft, 2016). Statistical analysis and processing was conducted using SPSS 20.0 (SPSS, Chicago, IL). Significance was defined as $\mathrm{P}<0.05$. Results were expressed as the mean \pm standard deviation (SD) for continuous normally distributed variables.

\section{Results}

Findings of this retrospective study on 54 cases of pulmonary aspergilloma surgically treated during the past 20 years are as follows: the mean age in this study was $46.3 \pm 7.8$, range (17 to 64 years). There were 39 males, resulting with a ratio of $2.6: 1$ to the female sex. The most common predisposing factor was tuberculosis. TB present in $57.4 \%$ of cases. The main presentation was recurrent hemoptysis in $70.4 \%$ of patients. Three patients with severe or massive hemoptysis were encountered during the whole period and were operated urgently. The radiological findings showed $34 / 54$ patients $62.9 \%$ showed positive meniscus sign while $16.6 \%$ showed a pulmonary nodule. There were 37 patients simple aspergilloma $68.5 \%$, while 17 patients showed a complex form (31.5\%). The left side was affected in $53.7 \%$ with predominant left upper lobe affection. The relevant laboratory tests revealed seropositive results in $64.8 \%$ and positive culture from protected bronchial lavage patients $(35.1 \%)$. In this series majority of patients underwent lobectomy, except 7 patients had wedge resection, the mean operative time was $140 \pm 15.6 \mathrm{~min}(75-220)$, two patients went to the ICU with packs to control apical adhesions bleeding and were re-explored 48 hours later. All patients were transferred to the ICU; the mean ICU stay was 48 hours, with next morning transfer to ward. Hospital stay ranged from 735 days with a mean of $9.5 \pm 6.4$ days. All patients after removal of the drains and free of complications were transferred to the medical ward to start their antifungal regimen according to the hospital policy and were followed up. Data analysis showed a follow up range of 1-5 years postprocedure.

Table 1. Preoperative patient data.

\begin{tabular}{ll}
\hline Age: & $\begin{array}{c}\text { Mean } 46.3 \pm 7.8 \\
\text { (range: } 17-64) \\
\text { Male no.: } .39\end{array}$ \\
Sex: & Female no.: $15(2.6: 1)$ \\
Predisposing factor: & \\
TB & $31 / 54(57.4 \%)$ \\
Bullous lung disease & $9 / 54(16.6 \%)$ \\
Bronchial asthma & $3 / 54(5.5 \%)$ \\
immunosuppression & $11 / 54(20.4 \%)$ \\
\hline
\end{tabular}




\begin{tabular}{|c|c|}
\hline \multicolumn{2}{|l|}{ Presentation } \\
\hline Hemoptysis & $38 / 54(70.4 \%)$ \\
\hline Shortness of breath & $40 / 54(74 \%)$ \\
\hline Cough and expectoration & $45 / 54(83.3 \%)$ \\
\hline \multicolumn{2}{|l|}{ Radiological findings } \\
\hline Simple Aspergilloma & $37 / 54(68.5 \%)$ \\
\hline Complex Aspergilloma & $17 / 54(31.5 \%)$ \\
\hline \multicolumn{2}{|l|}{ Site of lesion } \\
\hline Right side & $25 / 54(46.3 \%)$ \\
\hline Upper lobe & $18 / 54(33.3 \%)$ \\
\hline Middle lobe & $2 / 54(3.7 \%)$ \\
\hline Lower lobe & $5 / 54(9.3 \%)$ \\
\hline Left side & $29 / 54(53.7 \%)$ \\
\hline Upper lobe & $21 / 54(38.9 \%)$ \\
\hline Lower lobe & $8 / 54(14.8 \%)$ \\
\hline Consolidation & $6 / 54(11.1 \%)$ \\
\hline Nodule & $9 / 54(16.6 \%)$ \\
\hline Cavity with Halo sign & $34 / 54(62.9 \%)$ \\
\hline Destroyed lung with fibrosis & $5 / 54(9.3 \%)$ \\
\hline \multicolumn{2}{|l|}{ Laboratory findings } \\
\hline Positive Serological tests & $35 / 54(64.8 \%)$ \\
\hline Positive Bronchial lavage & $19 / 54(35.1 \%)$ \\
\hline \multicolumn{2}{|c|}{$\begin{array}{l}\text { During the follow up, we recorded prolonged air leak in } 16 \\
\text { patients }(29.6 \%) \text { that ranged from } 9-28 \text { days with a mean of } \\
13.5 \pm 5.4 \text { days. Wound infection was present in } 8 \text { patients } 14.8 \% \\
\text { of which one patient needed vacuum assisted device. Re- } \\
\text { exploration was needed in } 6 \text { patients }(11.1 \%) \text {, two were packed } \\
\text { and re-explored to remove packing. Cardiac arrhythmia was } \\
\text { encountered in } 4 \text { patients }(7.4 \%) \text {. In this series we encountered } 6 \\
\text { mortality ( } 12.3 \%) \text { cases. Two patients suffered respiratory } \\
\text { failure following severe chest infection and one patients } \\
\text { developed deep venous thrombosis (DVT) and massive } \\
\text { pulmonary embolism and } 3 \text { cases suffered circulatory failure. }\end{array}$} \\
\hline
\end{tabular}

During the follow up, we recorded prolonged air leak in 16 patients $(29.6 \%)$ that ranged from 9-28 days with a mean of $13.5 \pm 5.4$ days. Wound infection was present in 8 patients $14.8 \%$ of which one patient needed vacuum assisted device. Reexploration was needed in 6 patients $(11.1 \%)$, two were packed and re-explored to remove packing. Cardiac arrhythmia was encountered in 4 patients (7.4\%). In this series we encountered 6 mortality $(12.3 \%)$ cases. Two patients suffered respiratory failure following severe chest infection and one patients developed deep venous thrombosis (DVT) and massive pulmonary embolism and 3 cases suffered circulatory failure.

Table 2. Postoperative complications Complication.

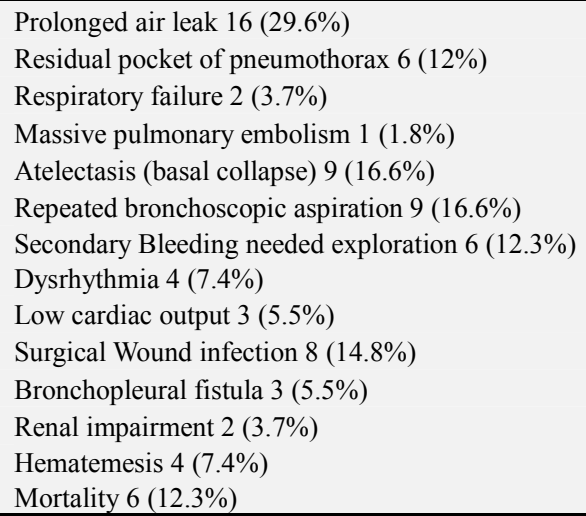

Table3. Two decades time; trends and outcomes.

\begin{tabular}{llll}
\hline Presentation Period & $\mathbf{1 9 9 6}-\mathbf{2 0 0 5}$ & $\mathbf{2 0 0 6}-\mathbf{2 0 1 5}$ & P value \\
\hline Mean age & $44.6 \pm 6.5$ & $48.7 \pm 8.9$ & 0.439 \\
Asymptomatic patients & $6 / 33(18.1 \%)$ & $9 / 21(42.8 \%)$ & 0.043 \\
Hemoptysis & $12 / 33(36.3 \%)$ & $5 / 21(23.8 \%)$ & 0.021 \\
Prevalence of TB & $15 / 33(45.4 \%)$ & $6 / 21(28.5 \%)$ & 0.017 \\
Need for thoracoplasty & $2 / 33(6.06 \%)$ & $0 / 21$ & 0.001 \\
Pleural space problems & $9 / 33(27.3 \%)$ & $7 / 21(33.3 \%)$ & 0.482 \\
Prolonged hospital stay & $12 / 33(36.3 \%)$ & $5 / 21(23.8 \%)$ & 0.716 \\
Secondary bleeding & $12 / 33(36.3 \%)$ & $3 / 21(14.2 \%)$ & 0.024 \\
Mortality & $4 / 33(12.1 \%)$ & $2 / 21(9.5 \%)$ & 0.024 \\
\hline
\end{tabular}

\section{Discussion}

The aspergilloma (commonly known as fungus ball or mycetoma) is composed of fungal hyphae, aggregates of inflammatory cells, fibrin threads, and destructed tissues debris, which usually hosted within a pre-existing lung cavitary lesion like tuberculosis, sarcoidosis, histoplasmosis or bullous emphysema and fibrotic lung disease. See figure 1 .

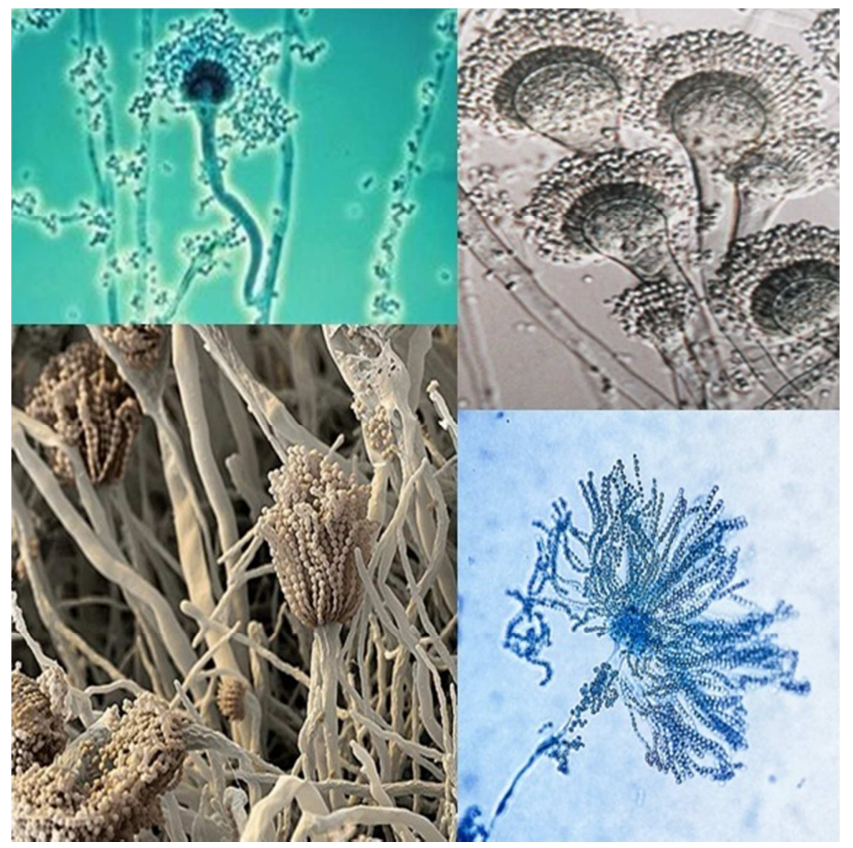

Figure 1. (top left \& right) represents electron microscopic images of Asperigullus fumigatus colony. (bottom left) 3 D electron microscopic image of cultured colony. [2].

Mycetoma is avascular and chronic inflammation means endarteritis oblitrans. These factors hinder the delivery of systemically administered antifungal agents into the cavity or the fungus ball; therefore, the logic is to resect. But still the surgery for mycetoma faces some technical difficulties and carries a high risk of complications if the patients were emaciated or under immunosuppression. [1, 2]

A dangerous symptom of mycetoma is recurrent hemoptysis. Minor degrees of hemoptysis can progress suddenly to massive hemoptysis in $30 \%$ of cases with an expected $25 \%$ mortality. [2] Air crescent sign is the cardinal radiological feature of the fungus ball of Aspergillosis. That is formed of condensed hyphae. The ball can be large or small, solitary or multiple. It may move when the patient's position changes or remain fixed, projecting into the cavity's lumen like a polyp. [2] Although it is often indolent with few or no symptoms in the beginning, the process frequently presents with massive hemoptysis, which can sometimes be fatal. Its differential diagnosis includes pulmonary Hydatid cyst, other fungi, blood clot or Rasmussen aneurysm in a tuberculous cavity, lung abscess with inspissated pus, Staphylococcal pneumonia, Nocardial infection, carcinoma of the lung, and lung gangrene or hematoma. [3]

The main presenting symptom in this series was hemoptysis, previous reports has recognized hemoptysis as 
high as $70 \%$. The radiological diagnosis of aspergilloma is positive when positive meniscus sign and mobile ball with patient position is confirmed. Chest CT scan are useful for diagnosis of simple and complex Aspergillosis as well as underlying lung parenchyma. Aspergilloma colonizes a preexisted cavitary space thus its localized lesion which doesn't extend to pleura. $[13,14]$ In the complex form, the process is more aggressive with more parenchymal destruction which reaches adjacent pleura. Pre-existing disease, most commonly tuberculosis is involved. [15] See figure 2. These patients are frequently sicker and have impaired pulmonary function tests due to widespread parenchymal involvement. Within the study, nine patients had a solitary pulmonary nodule and upon pathological report Aspergilloma was confirmed.
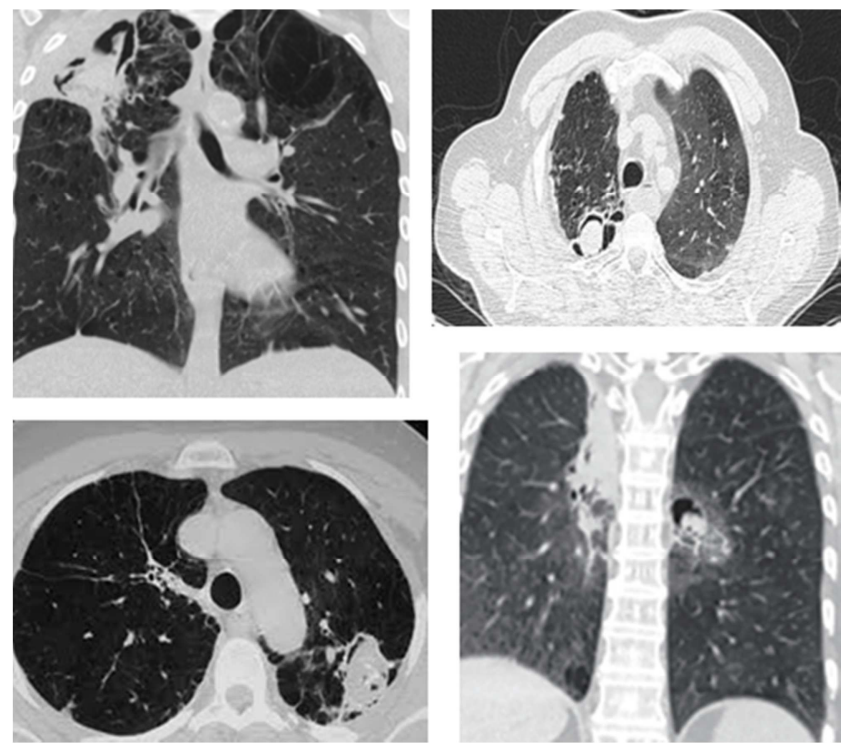

Figure 2. (2, a, b. top left \& right) CT chest sagittal and coronal views showing right apical cavitary lesion containing fungus ball. (c, $d$ bottom left \& right) left upper lobe asperagilloma seated at posterior segment.

Since antifungal has a poor penetration to thick cavity wall; hence resection is accepted if they are suitable candidates for surgery. VATS resections started to get a role in the surgical treatment of pulmonary fungal ball. [5] The surgery aims to preventing hemoptysis and increase survival. The most common surgery offered to Aspergilloma was lobectomy. Limited resections such as wedge or segmental resections became fewer due to pleural dissemination produced later on. [6-8]

Within our study, surgery was indicated in asymptomatic simple Aspergilloma in 9 cases were to achieve a diagnosis, where postoperative pathology results showed Aspergilloma. The fundamental question remains whether to operate on asymptomatic patients or not. It is our preferrence to perform surgery in asymptomatic simple Aspergilloma as a prophylaxis against $20 \%$ chance of severe hemoptysis that can be encountered during the course of the disease. [3, 7]

Patients with recurrent hemoptysis either with or without previous bronchial embolization were enrolled in our indication. Failed medical treatment and complex forms were indicated for resection as long as the residual lung showed good pulmonary function and patients have good functional performance. Complex form showed technical challenges with dense pleural adhesions, absence of interlobar fissure, fibrosis and inflammation at hilar vessels. These challenges reflected on postoperative complications as previously mentioned. See table 1. It is expected in this situation that patients suffer from prolonged air leaks, failure of lung expansion and postoperative bleeding. [1, 2] In our report, surgical procedures done were lobectomy in 43 cases, segmentaectomy in 7 cases, pneumonectomy in 2 cases and thoracoplasty in only 2 cases early in our series. Those patients were unlikely to survive conventional lobectomy, thus removal of fungus ball and collapsing lateral chest wall upon the residual cavity with acceptable postoperative outcome if compared to their debilitating status and the dangers of lobectomy. So, we still see thoracoplasty a viable solution but rarely used in modern practice. We done only 2 cases of pneumonectomy as we generally tend towards avoiding it whenever possible because of the fear of postpneumonectomy empyema. Aspergilloma surgery doomed to be tedious technically coupled with complicated postoperative course. $[5,8]$

We encountered 21/54 (39\%) postoperative complications. Most of them were due to prolonged air leaks and failure of lung expansion. Uncontrolled apical oozing was encountered in two patients who were transferred to the ICU packed and were re-explored and packs were removed 48 hours later. Almost all previous experiences have faced the same complications and were proportion to the extensive pathology and residual lung condition, complications ranged from $25 \%-70 \%$. $[1,9,10]$ Our mortality of $12.3 \%$ was to the lower side of most series. Although mortality was variable in different reports experiences, ranging from $1 \%$ and up to $43 \%$, this was due do the difference between each series in the percentage of high risk patients, complex Aspergillosis and pneumonectomies per study. [16, 17] Our reasonable mortality could be explained in view that $68.5 \%$ were simple Aspergilloma with localized disease and less challenging. Our 6/54 mortality patients were in the complex group. Two developed respiratory infection and respiratory failure. The third developed DVT in spite of anti-coagulation and was lost when he developed massive pulmonary embolism. Last two cases suffered circulatory failure.

In our study, morbidity like secondary bleeding was $36.3 \%$ in the first decade and $14.2 \%$ in the second decade, pleural problems were $27.3 \%$ and $33.3 \%$ respectively and hospital stay was 36.3 and $23.8 \%$ respectively. In fact, during first decade group, 9 patients had cumbersome pulmonary reexpansion either by lung fibrosis or loss of elasticity but none required thoracoplasty. In our report, we recorded an important decrease in postoperative morbidity, due to the less morbid profile through two decades periods after successful nation-wide control of tuberculosis. See table 3.

It is our own insight and recommendation to manage these patients in multidisciplinary team (MDT) approach, the decision for management is usually taken with chest 
physicians, intervention radiologist and the surgical team. Anti-fungal therapy at the perioperative period, as well as adjustment of preoperative co-morbidities as diabetes and nutrition status is paramount. The indication for anti-fungal therapy was directed to those with multiple cavities or those anticipated with the risk of pleural spillage during resection. Those with simple or contained Aspergilloma are in no need for antifungal therapy. In some patients with residual or other lung affection are given postoperative antifungal therapy to prevent recurrence. Other series adopted similar policy like ours. $[1,18]$

\section{Conclusion}

Surgery for pulmonary aspergillosis (fungus ball) remains the mainstay of management which offered beneficial satisfactory outcomes with an acceptable morbidity. The surgical options are few but over 2 decades, surgery for Asperigllosis became less invasive and less challenging might be due to nationwide eradication of tuberculosis. Though, mortality observed was predominantly seen in high risk patients with complex Aspergillosis. Although we present a small group of patients within this study, we emphasized the valuable lessons learned and complications discovered within this complex group. Consequently, we recommend MDT approach for the management of these patients with close follow-up. Despite being high risk surgery, results are good with low rate of recurrence.

\section{Ethical Approval and Responsibility}

Approval of the study protocol was granted by our Ethical Committee for research and treatment. The need for informed consent was waived due to the retrospective nature of the study. This work complies to Helsinki declaration. All of the authors have confirmed the preservation of confidentiality and respect of patients' rights in discretion and privacy.

\section{Availability of Data and Material}

Available on request.

\section{Disclosure}

The authors have nothing to disclose.

\section{Competing Interests}

The authors declare that they have no competing interests.

\section{Authors' Contributions}

All authors: 1) have made substantial contributions to conception, design, acquisition, analysis and interpretation of data; 2) have been involved in drafting the manuscript and revising it critically for important intellectual content; and 3) have given final approval of this version to be published.

\section{References}

[1] Kasprzyk M, Pieczyński K, Mania K, Gabryel P, Piwkowski C, and Dyszkiewicz W. Surgical treatment for pulmonary aspergilloma- early and long-term results. Kardiochir Torakochirurgia Pol. 2017 Jun; 14 (2): 99-103.

[2] Youssef C, and Widlus DM. Imaging diagnosis of aspergilloma. J Community Hosp Intern Med Perspect. 2012; 2 (1): 10 .

[3] Kurul IC, Demircan S, Yazici U, Altinok T, Topcu S, Unlü M. Surgical management of pulmonary aspergilloma. Asian CardiovascThorac Ann. 2004; 12 (4): 320-323.

[4] Greene R. The radiological spectrum of pulmonary aspergillosis. Medical Mycology Supplement 1 2005, 43, S147-S154.

[5] Ocakcioglu I, Ermerak NO, Yildizeli B. Uniportal Video-assisted Thoracoscopic Surgery for Pulmonary Aspergilloma: A Report of 5 Cases SurgLaparoscEndoscPercutan Tech. 2019; 29 (4): e37-e40.

[6] Regnard J, Icard P, Nicolosi M, Spagiarri L, Magdeleinat P, Jauffret B, et al. Aspergilloma: a series of 89 surgical cases. Ann Thorac Surg 2000; 69: 898-903.

[7] Park CK, Jheon S. Results of surgical treatment for pulmonary aspergilloma. Eur J Cardiothorac Surg. 2002; 21: 918-923.

[8] Soubani AO, Chandrasekar PH. The clinical spectrum of pulmonary aspergillosis. Chest. 2002; 121: 1988-1999.

[9] Akbari JG, Varma PK, Neema PK, Menon MU, Neelakandhan KS. Clinical profile and surgical outcome for pulmonary aspergilloma: a single center experience. Ann Thorac Surg. 2005; 80: 1067-1072.

[10] Massard G, Roeslin N, Wihlm JM, Dumont P, Witz JP, Morand G. Pleuropulmonary aspergilloma: clinical spectrum and results of surgical treatment. Ann Thorac Surg. 1992; 54: $1159-1164$.

[11] Al-Orainey I, Alhedaithy MA, Alanazi AR, Barry MA, and Almajid F. Tuberculosis incidence trends in Saudi Arabia over 20 years: 1991-2010. Ann Thorac Med. 2013 Jul-Sep; 8 (3): 148-152.

[12] Raveendran S. and Lu Z., CT findings and differential diagnosis in adults with invasive pulmonary aspergillosis. Radiology of Infectious Diseases 2018, (5) 14-25. https://doi.org/10.1016/j.jrid.2018.01.004.

[13] Massard G, Dabbagh A, Kessler R, Barsotti P, Roeslin N, Morand G. Pneumonectomy for chronic infection is a highrisk procedure. Ann Thorac Surg. 1996; 62: 1033-1038.

[14] Shiraishi Y, Katsuragi N, Nakajima Y, Hashizume M, Takahashi N, Miyasaka Y. Pneumonectomy for complex aspergilloma: is it still dangerous? Eur J Cardiothorac Surg. 2006; 29: 9-13.

[15] Rafferty P, Biggs BA, Crompton GK, Grant IW. What happens to patients with pulmonary aspergilloma? Analysis of 23 cases. Thorax. 1983; 38: 579-83.

[16] Franquet T., Mu'ller N., Giménez A., Guembe P., La Torre J., Bague S.; Spectrum of Pulmonary Aspergillosis: Histologic, Clinical, and Radiologic Findings. RadioGraphics 2001; 21: 825-837. 
[17] Sagan D, Goździuk K. Surgery for pulmonary aspergilloma in immunocompetent patients: no benefit from adjuvant antifungal pharmacotherapy. Ann Thorac Surg. 2010; 89: $1603-1610$.

[18] Farid S, Mohamed S, Devbhandari M, Kneale M, Richardson
M, Soon SY, Jones MT, Krysiak P, Shah R, Denning DW, Rammohan K. Results of surgery for chronic pulmonary Aspergillosis, optimal antifungal therapy and proposed high risk factors for recurrence-a National Centre's experience. J Cardiothorac Surg. 2013; 8: 180. 